\title{
Achat de médicaments sur Internet
}

\author{
En Suisse, de plus en plus de patients commandent des médicaments sur \\ Internet. Or, il faut savoir que cette pratique recèle des risques car les produits \\ distribués sur le Net peuvent être contrefaits, sans effet, voire dangereux pour \\ la santé.
}

Ruth Mosimann

Correspondance:

Ruth Mosimann

Swissmedic, Institut suisse des produits thérapeutiques Section Contrôle

des médicaments illégaux

Hallerstrasse 7

Case postale

CH-3000 Berne 9

Tél. 0313220472

Fax 0313220212

ruth.mosimann@swissmedic.ch

www.swissmedic.ch

\section{Importations de médicaments / mesures des autorités}

Les importations illégales sont bloquées à la douane suisse. Bien que les envois de médicaments soient très rarement déclarés comme tels, les douaniers découvrent régulièrement des importations illégales de ces produits. Après saisie en douane, Swissmedic lance une procédure administrative entraînant la perte (généralement sous la forme d'une destruction) des médicaments. Les coûts liés à cette procédure se montent à 300 francs au moins, ces frais devant être supportés par le client en Suisse.

La loi sur les produits thérapeutiques autorise une importation en toute légalité de médicaments en petites quantités (besoins pour un mois) pour un usage personnel. Cette disposition conçue à l'origine pour les touristes entrant en Suisse est de plus en plus utilisée par nos compatriotes pour se procurer, via Internet, des médicaments soumis à prescription médicale en provenance de l'étranger. Une action récente a ainsi révélé que $27 \%$ de tous les colis postaux importés d'Inde et $16 \%$ de ceux provenant de Thaillande contenaient des médicaments. $40 \%$ de ces importations par des particuliers respectaient les quantités admises par la loi.

\section{Qualité}

La qualité des médicaments commandés sur Internet est très variable. On ne peut cependant exclure le risque qu'il s'agisse de contrefaçons. Les contrefaçons de médicaments sont des produits présentant des données sciemment erronées quant à leur composition et/ou leur provenance. Il arrive souvent que les médicaments contrefaits ne contiennent aucun principe actif, ne recèlent pas le bon principe actif ou soient trop ou insuffisamment dosés. En outre, ils ont été fabriqués dans des conditions non contrôlées et n'ont donné lieu à aucune étude clinique.

Certains groupes de médicaments font également l'objet d'imitations. Ces imitations illégales ne cherchent pas à copier directement une préparation originale dans son conditionnement et contiennent souvent le même principe actif ou ont une présentation similaire à celle de l'original quant à l'indication.

\section{Effets indésirables de médicaments illégaux}

Un certain nombre de médicaments contre l'impuissance apparus cette année en Asie contenaient non pas des principes actifs stimulant l'érection mais une dose particulièrement élevée de glibenclamide, un antidiabétique oral. Les autorités de Singapour ont constaté ces contrefaçons suite à l'annonce d'un effet indésirable de ces produits. Un patient était en effet décédé suite à une hypoglycémie sévère ayant nécessité son hospitalisation. La Suisse a elle aussi découvert un cas de contrefaçon dangereuse pour la santé il y a quelques semaines: le «Powertabs», qui était présenté comme un médicament exclusivement à base de plantes contre l'impuissance. Or, les analyses réalisées par Swissmedic ont révélé que le «Powertabs» contenait en réalité un principe actif apparenté au sildénafil, n'ayant fait nulle part l'objet d'une autorisation.

\section{Figure 1}

Produits contre les troubles érectiles importés illégalement: contrefaçons (à gauche) et imitations (à droite).

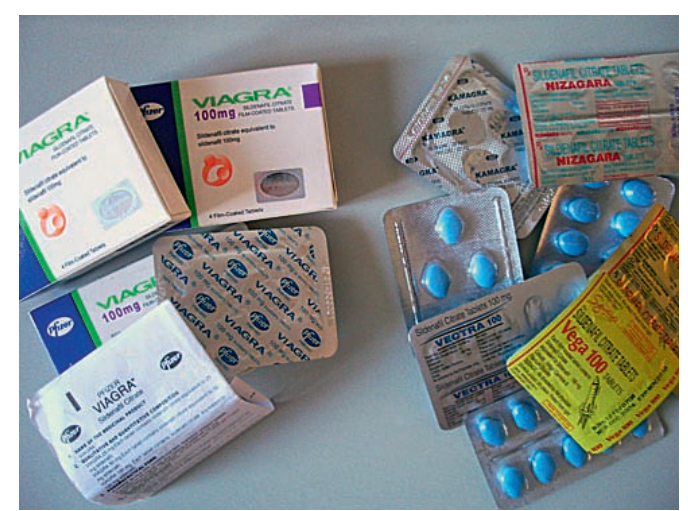


Eu égard à la quantité relativement importante de médicaments achetés sur Internet par des particuliers, Swissmedic en appelle au corps médical pour signaler les médicaments importés qui seraient à l'origine d'effets indésirables ou d'incidents inattendus chez des patients. Veuillez donc utiliser le formulaire jaune d'annonce d'effets indésirables de médicaments, même lorsque cet effet indésirable provient d'un médicament non autorisé ou importé de façon illégale. Ce formulaire est disponible sur le site Web de Swissmedic (www.swissmedic.ch, sous Surveillance du marché $\rightarrow$ Pharmacovigilance $\rightarrow$ Répertoire général $\rightarrow$ Formulaire d'annonce) et en annexe du Compendium suisse des médicaments ou peut être commandé auprès de Swissmedic au tél. 0313220223.

\section{Campagne de sensibilisation du public}

Malgré le combat mené par Swissmedic contre les importations illégales et sa collaboration toujours plus étroite avec des autorités étrangères pour lutter contre les pharmacies en ligne, il est impossible de tarir à la source les flux de médicaments d'origines douteuses. Dans ce contexte, il est donc essentiel que la population suisse prenne conscience qu'elle ne doit pas commander de médicaments sur Internet.

Swissmedic met régulièrement en garde dans des communiqués de presse contre des médicaments dangereux. Un guide actualisé sur les risques et les bases légales relatives à la commande de médicaments sur Internet a ainsi été mis en ligne cette année sur le site de Swissmedic. En outre, Swissmedic a initié, de concert avec Interpharma, une campagne placée sous l'égide de la plate-forme «STOP À LA PIRATERIE» (STOP PIRACY). Cette campagne, diffusée par différents médias, se terminera sur la journée d'action
«STOP PIRACY DAY» le 25 octobre 2008, durant laquelle les particuliers pourront notamment faire contrôler dans plus de 500 pharmacies suisses l'authenticité de leurs médicaments commandés sur Internet.

\section{Figure 2}

Logo de la journée d'action dans les pharmacies.

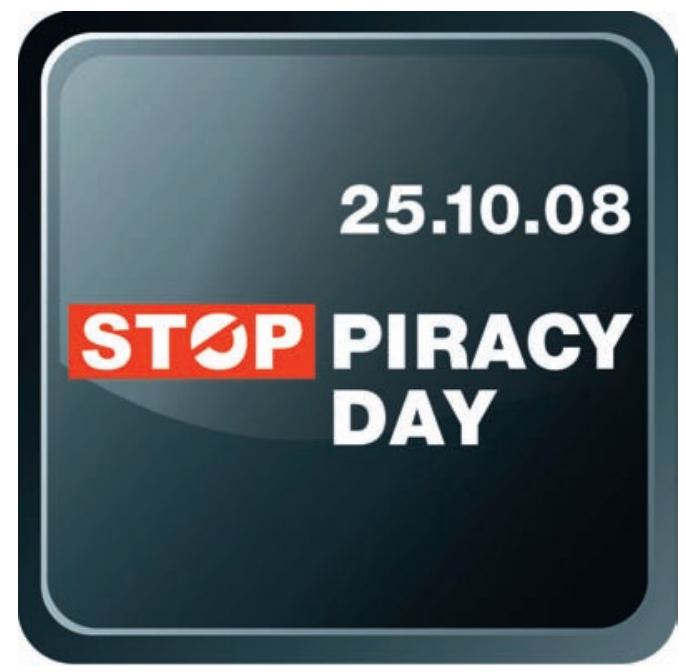

\section{Importations de médicaments}

Swissmedic estime qu'au moins 50000 colis sont importés illégalement tous les ans en Suisse par des particuliers.

En collaboration avec les douanes, Swissmedic saisit les envois illégaux, dans lesquels on trouve souvent:

- des produits contre l'impuissance;

- des anabolisants;

- des produits amaigrissants;

- d'autres préparations soumises à ordonnance. 